\title{
Soft tissue sarcoma in neurofibromatosis type 1: A rare case of malignant peripheral nerve sheath tumor of the skin
}

\author{
Young Hun Chung ${ }^{1}$, Jin Woo Jang ${ }^{2}$, Jae Young Cho ${ }^{2}$ \\ ${ }^{1}$ Department of Plastic Surgery, Kyung Hee University Graduate School, Seoul; ${ }^{2}$ Department of Plastic Surgery, Kyung Hee University \\ Medical Center, Seoul, Korea
}

Malignant peripheral nerve sheath tumor (MPNST) is a rare and often aggressive soft tissue sarcoma originating from the sheaths of peripheral nerves. Approximately 50\% of MPNSTs occur in patients with neurofibromatosis (NF). These tumors often present as deep soft tissue lesions, arising from the nerve plexuses of the extremities or from the nerves extending from the trunk. They rarely occur in the skin, especially in patients with NF. Herein, we report our experience with an MPNST of the skin in a patient with NF.

Keywords Neurofibromatosis 1 / Nerve sheath neoplasm / Skin neoplasm
Correspondence: Jae Young Cho Department of Plastic Surgery, Kyung Hee University Medical Center, 23 Kyungheedae-ro, Dongdaemun-gu, Seoul 02447, Korea

Tel: $+82-2-958-8431$

Fax: +82-2-963-5638

E-mail: jelly2750@daum.net

Received: October 16, $2019 \bullet$ Revised: November 6, 2019 • Accepted: November 6, 2019

pISSN: 2234-6163 • elSSN: 2234-6171 • https://doi.org/10.5999/aps.2019.01529• Arch Plast Surg 2020;47:92-96

\section{INTRODUCTION}

Neurofibromatosis (NF), a polymorphic form of neurofibroma, is an autosomal dominant disorder with a prevalence of one in 4,000 people [1-3]. It is caused by a defect or mutation of a tumor suppressor gene located on chromosome 17 [3]. The most common type of NF is NF type 1 (NF-1), the main features of which include café au lait spots, multiple neurofibromas, Lisch nodules, and specific osseous dysplasia [2,3]. Although malignancies associated with NF-1 are rare, the condition is associated with an increased risk of malignant peripheral nerve sheath tumors (MPNSTs), optic gliomas, and leukemia.

An MPNST is a spindle cell sarcoma that originates from the cells of peripheral nerves and nerve sheaths [4]. It is a rare tumor, accounting for $2 \%$ of all sarcomas. It can be diagnosed if a histopathological examination shows distinct features of Schwann cell differentiation [5]. Approximately 50\% of MPNSTs develop in patients with NF-1 [4]. MPNSTs generally appear as deep tissue masses in the extremities or the trunk. They arise from nerve plexuses in the extremities, such as the brachial or lumbar plexus, or from nerves extending from the trunk. MPNSTs rarely occur in the skin, particularly in patients with NF-1 [6,7]. Herein, we report a case of an MPNST of the skin in a patient with NF-1, along with a review of the literature.

\section{CASE}

A 77-year-old woman presented to our clinic with a mass on her left flank that had grown rapidly over 3 months. The patient had been diagnosed with NF-1 45 years ago. She also had asthma, hypertension, and atrial fibrillation, and had undergone percutaneous nephrostomy insertion in her left kidney to treat a urethral obstruction.

Clinical examination revealed a protruding firm nodule arising from a dermal neurofibroma in the patient's left flank (Fig. 1). An ulcerated lesion with bloody discharge was present in the 
most prominent area, and the patient reported experiencing pain and tenderness. Magnetic resonance imaging revealed a well-enhancing mass $(3.9 \times 2.5 \times 3.4 \mathrm{~cm})$ with a broad base along the dermis, with a round bulging contour on the lateral side. The mass showed intermediate-to-high $\mathrm{T} 1$ signals with relatively homogeneous enhancement (Fig. 2). Histological examination showed infiltration of tumor tissue into the dermis, which was consistent with the appearance of interlacing bundles of neoplastic spindle cells with marked pleomorphism. The hypercellular areas consisted of clusters and sheets of epithelioid round cells admixed with fascicles of hyperchromatic spindled

\section{Fig. 1. Clinical findings of the tumor \\ A polypoid, prominent, and enlarged dermal neurofibroma with an unencapsulated lesion in the center was observed on the patient's left flank. She had multiple dermal neurofibromas on her body. A nephrostomy catheter was present on the lower side of the lesion.}

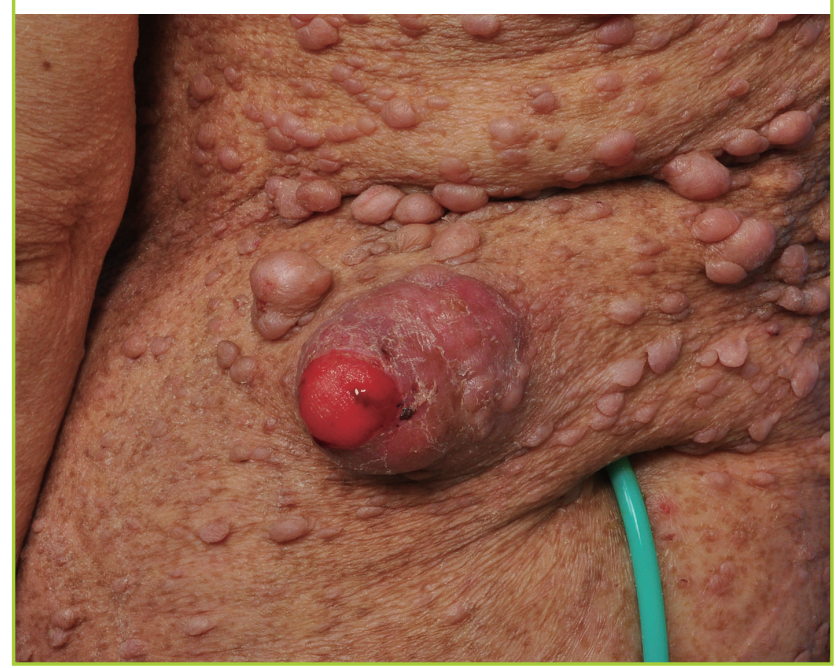

cells. No lymphatic or vascular invasion was identified (Fig. 3). Immunohistochemical staining was positive for S-100 protein in the tumor cell cytoplasm (Fig. 4).

After diagnosis of the mass as a cutaneous epithelioid MPNST (CE-MPNST), complete surgical excision was planned. With the patient under general anesthesia, the grossly visible tumor was completely excised with lateral and deep resection margins of $30 \mathrm{~mm}$ and $15 \mathrm{~mm}$, respectively (Fig. 5). After frozen biopsy to confirm the absence of malignancy, additional undermining was performed to elevate the surrounding subcutaneous tissue. A bilateral fasciocutaneous flap was elevated and advanced and was then closed layer by layer. The wound healed well after surgery, and no signs of local recurrence or metastasis were ob-

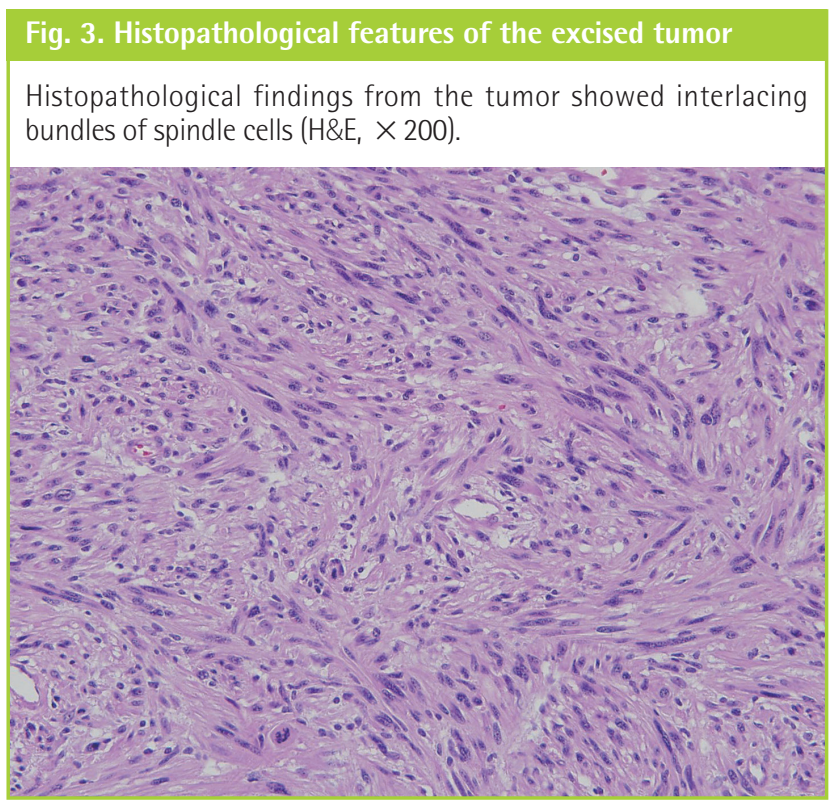

\section{Fig. 2. Magnetic resonance images of the tumor}

Magnetic resonance imaging showed a mass of $3.9 \times 2.5 \times 3.4 \mathrm{~cm}$ with a relatively clear boundary, bulging along the dermis on the left flank. It showed intermediate-to-high T1 signals with relatively homogeneous enhancement. (A) T1 axial view. (B) T1 coronal view.
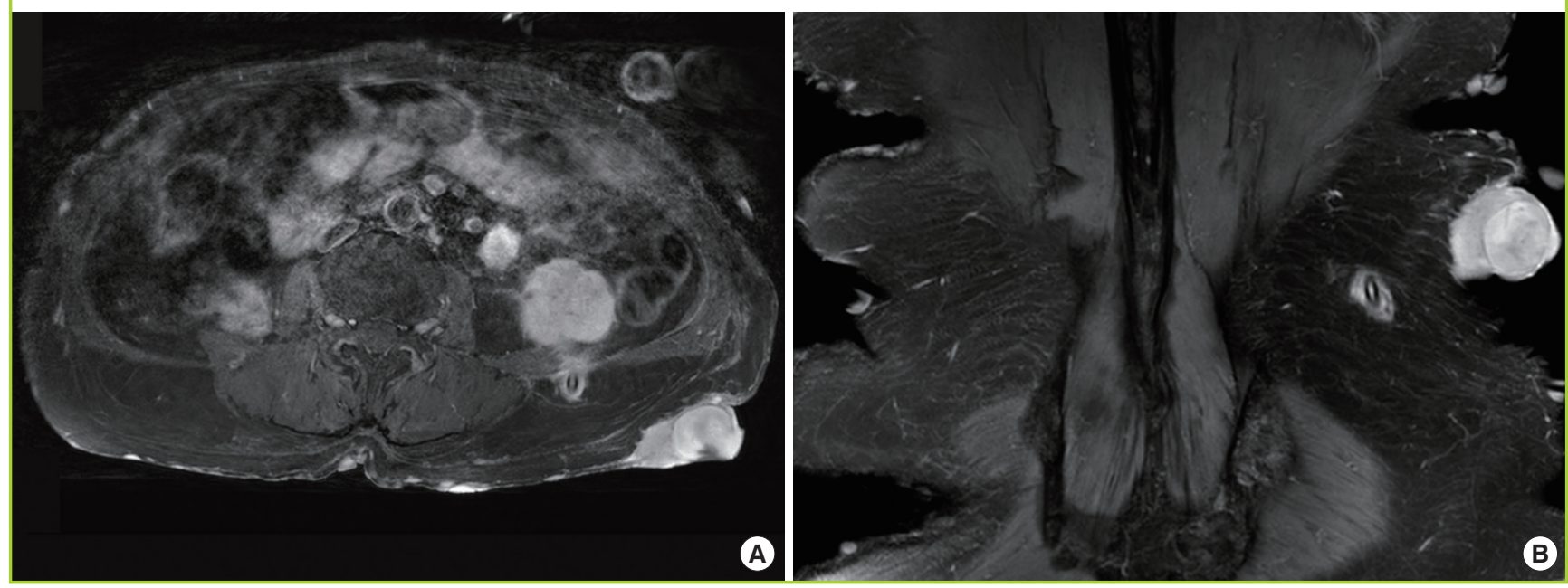


\section{Fig. 4. Immunohistochemical features of the excised tumor \\ Immunohistochemical staining for S-100 protein showed positive immunoreactivity $(\times 200)$.}

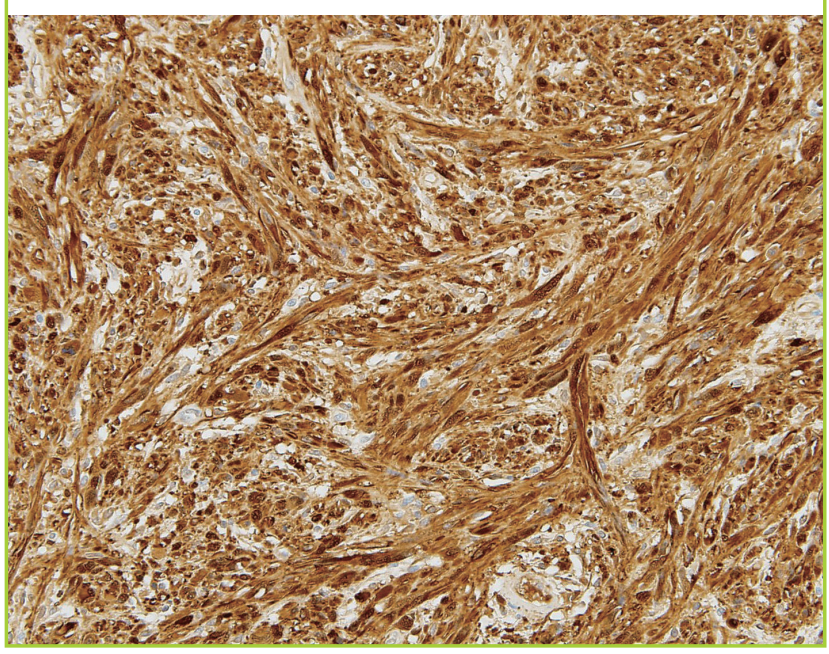

\section{Fig. 5. Gross findings of the excised tumor}

Gross findings of the excised tumor, including the lesion and 30$\mathrm{mm}$ and 15-mm lateral and deep margins, respectively.

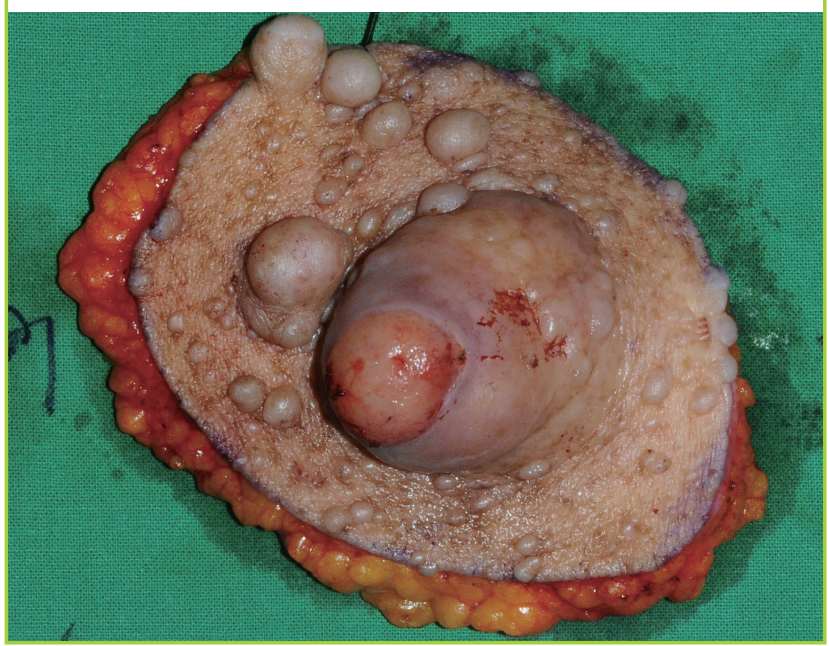

served 15 months later (Fig. 6).

This study was approved by the Institutional Review Board of Kyung Hee University Medical Center on October 4, 2019 (IRB No. 2019-09-041) and was performed in accordance with the principles of the Declaration of Helsinki. Informed consent was obtained from the patient.

\section{DISCUSSION}

MPNSTs are generally associated with the nerve plexuses, which are located deep in the subcutaneous tissue. MPNSTs of the skin are rarely reported $[6,7]$ and are termed cutaneous
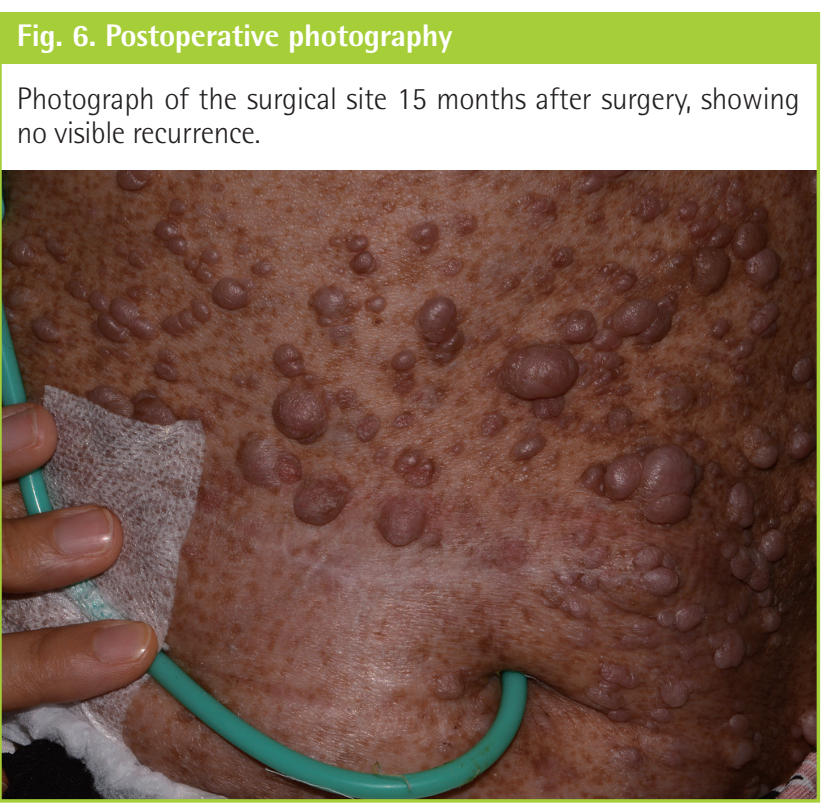

MPNST (C-MPNST) [4]. Histologically, mitotic activity in CMPNSTs is markedly increased. Organized cell growth patterns, consisting of densely packed spindle cells arranged in cellular fascicles, are observed without an extracellular matrix $[3,5]$. The immunohistochemical findings are notable; immunostaining for S-100 protein, which is mainly found in cells of the central and peripheral nervous systems, is positive in approximately $50 \%$ of MPNSTs, while S-100 is rarely present in other spindle cell tumors $[3,5,8]$. Similarly, C-MPNSTs test strongly positive for S-100 protein $[4,6]$. Staining for Ki-67 may be helpful in diagnosing C-MPNST; although Ki-67 is present in $<5 \%$ of atypical neurofibroma cells, it is more commonly found in MPNSTs [7].

C-MPNSTs are divided into two subtypes, which differ according to the location of occurrence and their immunohistochemical features. Cutaneous conventional MPNSTs (CCMPNSTs) generally occur in the head and neck or the limbs and are characterized by slow growth over a relatively long period, followed by a period of rapid growth. Under immunostaining, CC-MPNST cells test negative for S-100 protein in approximately half of patients, and those that do test positive typically exhibit focal positivity [4]. CE-MPNSTs, of which our case was an example, frequently occur in the lower extremities or trunk, with no distinctive clinical features, and appear as nontender discrete nodules. In addition, immunohistologically, CEMPNSTs exhibit strong, diffuse S-100 positivity [4]. CEMPNST has a more aggressive clinical course than CC-MPNST [9]. A recent study found that one-third of patients with CEMPNST died of disseminated disease in a follow-up period that ranged from 24 to 100 months [10]. 
This case had three major findings. First, C-MPNST of the skin occurred in a patient with NF-1. In general, the association between MPNST and NF-1 is well known [1,2]. Several studies have reported the occurrence of MPNST in NF-1 patients in Korea $[3,8,9]$. However, previous studies showed only a rare association between NF-1 and C-MPNST $[4,7,11]$. Only one case of C-MPNST of the skin in NF-1 has been reported worldwide [12].

Second, the C-MPNST in our patient appears to have arisen via malignant transformation from dermal neurofibroma. Previous studies have reported that superficial lesions derived from dermal neurofibromas or small peripheral nerve branches are generally asymptomatic and are relatively unlikely to transform into malignant neoplasms [5]. MPNSTs usually develop from preexisting plexiform neurofibromas, which are rare in the general population. Plexiform neurofibromas have the same histological structure as dermal neurofibromas, but they have a more extensive extracellular matrix and are often better vascularized. They expand along the length of a nerve and can infiltrate nerve roots and nerve plexuses. Until now, no evidence has indicated that dermal neurofibromas or flat superficial plexiform neurofibromas can transform into malignancies [12]. However, our patient's physical findings suggest that the C-MPNST was mixed with the surrounding dermal neurofibroma. These findings suggest that the existing dermal neurofibroma has been maligned as C-MPNST.

Third, previous reports have shown that conventional MPNST and C-MPNST are well-encapsulated and do not invade or adhere to surrounding tissues $[3,12,13]$. However, the mass found in our patient contained an unencapsulated lesion and displayed a broad base along the dermis on magnetic resonance imaging.

Complete surgical excision is a treatment option in cases of CMPNST [1]. Response to chemotherapy and radiotherapy is known to be poor, and chemotherapy can be used only for metastatic diseases $[2,5]$. Our patient underwent surgery, after which no adjuvant therapy was administered. The most important prognostic factor is tumor size, which is typically $>5 \mathrm{~cm}$ at presentation. Other factors include tumor grade, surgical margin, local recurrence, histological malignancy, cell polymorphism, and mitotic activity $[3,5]$.

Previous studies have reported that a clear surgical margin is the most important factor involved in the survival of patients with C-MPNST $[14,15]$. Accordingly, our patient survived the 15-month follow-up period without recurrence or metastasis after complete resection with a negative margin. This suggests that conventional management can be applied in cases similar to that of our patient.

According to previous reports, malignant transformation of dermal neurofibromas into MPNST is rare. However, as our case shows, it cannot be ruled out completely. If a dermal neurofibroma grows rapidly and surface changes are observed, clinicians must consider the possibility of malignancy.

\section{NOTES}

\section{Conflict of interest}

No potential conflict of interest relevant to this article was reported.

\section{Ethical approval}

The study was approved by the Institutional Review Board of Kyung Hee University Medical Center (IRB No. 2019-09-041) and performed in accordance with the principles of the Declaration of Helsinki. Written informed consent was obtained.

\section{Patient consent}

The patient provided written informed consent for the publication and the use of her images.

\section{Author contribution}

Conceptualization: Cho JY. Formal analysis: Chung YH, Jang JW. Methodology: Chung YH. Project administration: Cho JY. Visualization: Jang JW. Writing - original draft: Chung YH. Writing - review \& editing: Chung YH, Cho JY.

\section{ORCID}

Young Hun Chung https://orcid.org/0000-0001-8032-036X

Jin Woo Jang https://orcid.org/0000-0002-5073-8666

\section{REFERENCES}

1. Evans DG, Baser ME, McGaughran J, et al. Malignant peripheral nerve sheath tumours in neurofibromatosis $1 . \mathrm{J}$ Med Genet 2002;39:311-4.

2. Ferner RE, Gutmann DH. International consensus statement on malignant peripheral nerve sheath tumors in neurofibromatosis. Cancer Res 2002;62:1573-7.

3. Kim DH, Lee SS, Lee SH, et al. A case of malignant peripheral nerve sheath tumor of the neck associated with neurofibromatosis type I. Korean J Otorhinolaryngol-Head Neck Surg 2010;53:657-60.

4. Luzar B, Falconieri G. Cutaneous malignant peripheral nerve sheath tumor. Surg Pathol Clin 2017;10:337-43.

5. Farid M, Demicco EG, Garcia R, et al. Malignant peripheral nerve sheath tumors. Oncologist 2014;19:193-201.

6. George E, Swanson PE, Wick MR. Malignant peripheral 
nerve sheath tumors of the skin. Am J Dermatopathol 1989; 11:213-21.

7. Thomas C, Somani N, Owen LG, et al. Cutaneous malignant peripheral nerve sheath tumors. J Cutan Pathol 2009; 36:896-900.

8. Park CH, Kim HS, Lee JH, et al. A case of malignant peripheral nerve sheath tumor in nasal cavity. Korean J Otorhinolaryngol-Head Neck Surg 2008;51:376-9.

9. Jo VY, Fletcher CD. Epithelioid malignant peripheral nerve sheath tumor: clinicopathologic analysis of 63 cases. Am J Surg Pathol 2015;39:673-82.

10. Luzar B, Shanesmith R, Ramakrishnan R, et al. Cutaneous epithelioid malignant peripheral nerve sheath tumour: a clinicopathological analysis of 11 cases. Histopathology 2016;68:286-96.

11. Inoue T, Kuwashiro M, Misago N, et al. Superficial malig- nant peripheral nerve sheath tumor arising from diffuse neurofibroma in a neurofibromatosis type 1 patient. J Dermatol 2014;41:631-3.

12. Dietrich V, Schaller J, Kunze J. Cutaneous malignant peripheral nerve sheath tumor in neurofibromatosis type 1 . Hautarzt 2009;60:830-3.

13. Lee HS, Lee CH, Jin SM, et al. A case of malignant peripheral nerve sheath tumor in parapharyngeal space. Korean J Otorhinolaryngol-Head Neck Surg 2012;55:181-4.

14. Hannah CE, Moye MS, Liu V, et al. Cutaneous metastases of malignant peripheral nerve sheath tumor: ineffectiveness of intralesional methotrexate. JAAD Case Rep 2018;4:716-8.

15. Thway K, Fisher C. Malignant peripheral nerve sheath tumor: pathology and genetics. Ann Diagn Pathol 2014;18: 109-16. 